\title{
APPLICATIONS OF KAUTZ MODELS IN SYSTEM IDENTIFICATION
}

\author{
P. LINDSKOG \\ Dept. of Electrical Engineering \\ Linköping University \\ S-581 83 Linköping, Sweden \\ Email: lindskog@isy.liu.se
}

\author{
B. WAHLBERG \\ Dept. of Signals, Sensors and Systems \\ Royal Institute of Technology \\ S-100 44 Stockholm, Sweden \\ Email: bo@elixir.e.kth.se
}

\begin{abstract}
FIR, ARX or AR model structures can be used to describe many industrial processes. Simple linear regression techniques can be applied to estimate such models from experimental data. However, for low signal to noise ratios in combination with transfer function poles and noise model zeros close to the unit circle, a large number of model parameters are needed to generate adequate models. The Kautz model structure generalizes FIR, ARX and AR models. By using a priori knowledge about the dominating time constants and damping factors of the system, the model complexity is reduced, and the linear regression structure is retained. The objective of this contribution is to study an industrial example, where Kautz models have distinct advantages. The data investigated corresponds to aircraft flight flutter, which is a state when an aircraft component starts to oscillate.
\end{abstract}

Key Words. Modeling, system identification, parameter estimation, aircraft modeling, Kautz functions.

\section{INTRODUCTION}

To describe the behavior of real systems using dynamical models is of outmost importance in many fields of science. System identification deals with the problem of constructing dynamical models from experimental data. By far, the most used model in system identification is the ARX model

$$
A(q) y(t)=B(q) u(t)+e(t),
$$

where $u(t)$ is the input signal, $y(t)$ is the output signal, the noise process $\{e(t)\}$ is assumed to be a sequence of independent stochastic variables and

$$
\begin{aligned}
& A(q)=1+\bar{a}_{1} q^{-1}+\ldots+\bar{a}_{n a} q^{-n a} \\
& B(q)=q^{-n k}\left[\bar{b}_{1}+\ldots+\bar{b}_{n b} q^{-(n b-1)}\right] .
\end{aligned}
$$

Here $q^{-1}$ denotes the shift operator: $q^{-1} u(t)=$ $u(t-1)$. In case there are no external inputs, $B(q)=0$, an AR time-series model structure is obtained. By taking $A(q)=1$ the result is a FIR model structure.

The ARX, FIR and AR model structures have many nice properties and provide useful models in many applications. However, they also have some drawbacks:

Consider the following model of a general stable system

$$
y(t)=G(q) u(t)+H(q) e(t) .
$$

The connection to an ARX model is

$$
\begin{aligned}
& A(q) \approx H(q)^{-1}=1+\sum_{k=1}^{\infty} \bar{a}_{k} q^{-k}, \\
& B(q) \approx H(q)^{-1} G(q)=\sum_{l=1}^{\infty} \bar{b}_{l} q^{-l} .
\end{aligned}
$$

To simplify the discussion assume that $H(q)=1$. The FIR approximation of $B(q)$ can then be viewed as a truncation of the Laurant series expansion of $G(q)$. The number of parameters, $n b$, needed to obtain an useful FIR description is thus determined by the rate of decrease of the impulse response. Systems with poles close to the unit circle have slowly decreasing impulse responses. Consequently, high order FIR models are then required to obtain sufficient flexible model descriptions.

For the general ARX model structure noise model zeros of $H(q)$ close to the unit circle are also a problem, since $A(q)$ and $B(q)$ can be viewed as truncations of the Laurant series expansion of $H(q)^{-1}$ and $H(q)^{-1} G(q)$, respectively.

\section{KAUTZ MODEL STRUCTURES}

To circumvent the problems with ARX models, the delay operator can be replaced by so-called discrete Laguerre or Kautz filters. The Laguerre 
filter is defined as

$$
\begin{aligned}
L_{k}(q, a) & =\frac{\sqrt{\left(1-a^{2}\right)}}{q-a}\left[\frac{1-a q}{q-a}\right]^{k-1}, \\
a & \in \mathbb{R}, \quad|a|<1, \quad k \geq 1,
\end{aligned}
$$

and the definition of the Kautz filter is

$$
\begin{gathered}
\Psi_{k}(q, b, c)= \\
\left\{\begin{array}{l}
\frac{\sqrt{1-c^{2}}(q-b)}{q^{2}+b(c-1) q-c}\left[\frac{-c q^{2}+b(c-1) q+1}{q^{2}+b(c-1) q-c}\right]^{\frac{(k-1)}{2}} \\
\frac{\sqrt{\left(1-c^{2}\right)\left(1-b^{2}\right)}}{q^{2}+b(c-1) q-c} \\
k \text { edd }
\end{array}\left[\frac{-c q^{2}+b(c-1) q+1}{q^{2}+b(c-1) q-c}\right]^{\frac{(k-2)}{2}}\right. \\
\quad b, c \in \mathbb{R}, \quad|b|<1, \quad|c|<1, \quad k \geq 1
\end{gathered}
$$

The Laguerre filters are appropriate for welldamped systems, whereas Kautz filters preferably can be used for more resonant ones. To obtain adequate low order models one must choose $a$ or $b$ and $c$ to correspond to the dominating pole (zero for time-series) of the true system. Observe that by taking $a=0$ or $b=c=0$ the original base functions $\left\{q^{-k}\right\}$ are obtained. Further properties of these filters, motivations and applications in system identification are given in Wahlberg (1991a) and Wahlberg (1991b).

Given the new base functions a Laguerre/Kautz model structure is defined as

$$
\begin{aligned}
{\left[1+\sum_{k=1}^{n a} \bar{a}_{k} \mathcal{A}_{k}(q)\right] y(t) } & = \\
& q^{-n k}\left[\sum_{k=1}^{n b} \bar{b}_{k} \mathcal{B}_{k}(q)\right] u(t)+e(t),
\end{aligned}
$$

where

$\mathcal{A}_{k}(q)=\mathcal{B}_{k}(q)=\left\{\begin{array}{l}L_{k}(q, a) \text { in the Laguerre case, } \\ \Psi_{k}(q, b, c) \text { in the Kautz case. }\end{array}\right.$

Note the distinction between the unknown model parameters $\bar{a}_{k}$ and $\bar{b}_{k}$ and the expected time constants specified by the filter coefficients $a, b$ and $c$. As for the ARX case this model structure can be rewritten in the linear regression form

$$
\begin{aligned}
y(t)= & \varphi(t)^{\prime} \theta+e(t) \\
\varphi(t)= & {\left[-\mathcal{A}_{1}(q) y(t) \ldots-\mathcal{A}_{n a}(q) y(t)\right.} \\
& \left.\mathcal{B}_{1}(q) u(t-n k) \ldots \mathcal{B}_{n b}(q) u(t-n k)\right]^{\prime}, \\
\theta= & {\left[\begin{array}{ll}
\bar{a}_{1} \ldots \bar{a}_{n a} & \bar{b}_{1} \ldots \bar{b}_{n b}
\end{array}\right]^{\prime} . }
\end{aligned}
$$

Estimation of $\theta$ can now be done using the standard least squares method.

Due to the linear properties of these structures one can handle multiple time constants by cascading a number of Laguerre and Kautz models. Such a general model structure can thus be defined as

$$
\begin{aligned}
{[1+} & \sum_{i=1}^{m^{\mathcal{L}}} \sum_{k=1}^{n a_{i}^{\mathcal{L}}} \bar{a}_{i, k}^{\mathcal{L}} L_{k}\left(q, a_{i}\right)+ \\
& \left.\sum_{i=1}^{m^{\mathcal{K}}} \sum_{k=1}^{n a_{i}^{\mathcal{K}}} \bar{a}_{i, k}^{\mathcal{K}} \Psi_{k}\left(q, b_{i}, c_{i}\right)\right] y(t)= \\
& {\left[\sum_{i=1}^{m^{\mathcal{L}}} \sum_{k=1}^{n b_{i}^{\mathcal{L}}} \bar{b}_{i, k}^{\mathcal{L}} L_{k}\left(q, a_{i}\right) q^{-n k_{i}^{\mathcal{L}}}+\right.} \\
& \left.\sum_{i=1}^{m^{\mathcal{K}}} \sum_{k=1}^{n b_{i}^{\mathcal{K}}} \bar{b}_{i, k}^{\mathcal{K}} \Psi_{k}\left(q, b_{i}, c_{i}\right) q^{-n k_{i}^{\mathcal{K}}}\right] u(t)+e(t),
\end{aligned}
$$

where superscript $\mathcal{L}(\mathcal{K})$ refers to the Laguerre (Kautz) part of the system and $m$ is the number of cascaded models. Observe that this model structure still can be written in a linear regression form.

To attain a feasible low order model the dominating time constants of the real system must roughly be known. This a priori knowledge can, for instance, be gained through pilot step response tests or via spectral analysis. The time constants will of course only be approximately known, but based on these preliminary experiments it is possible to estimate the dominating time constants by adopting special non-linear search strategies (in this case a Gauss-Newton scheme). Notice that by using a Newton algorithm only local convergence can be guaranteed, meaning that the "optimal" time constants not necessarily are found. However, once the base functions are fixed the least squares method will always generate a global optimal solution with respect to the chosen base functions. This procedure for finding suitable base functions is followed in the next section.

\section{AIRCRAFT FLIGHT FLUTTER}

Flight flutter is when an aircraft component at a specific airspeed starts to oscillate. Above this critical or "flutter" speed disturbances will grow uncontrolled until they are finally limited by nonlinearities, whereas at lower speeds disturbances will be damped out. The experiments are usually carried out by attaching special transducers to the airframe, in this case to the wings, thereby introducing artificial mechanical vibrations. When flying at constant speed data is often analyzed offline, giving information about whether to allow the aircraft to fly faster or not. To a certain extend the flutter behavior is therefore a measure of the maximum performance of the aircraft.

Data gained during flight flutter tests is typically 

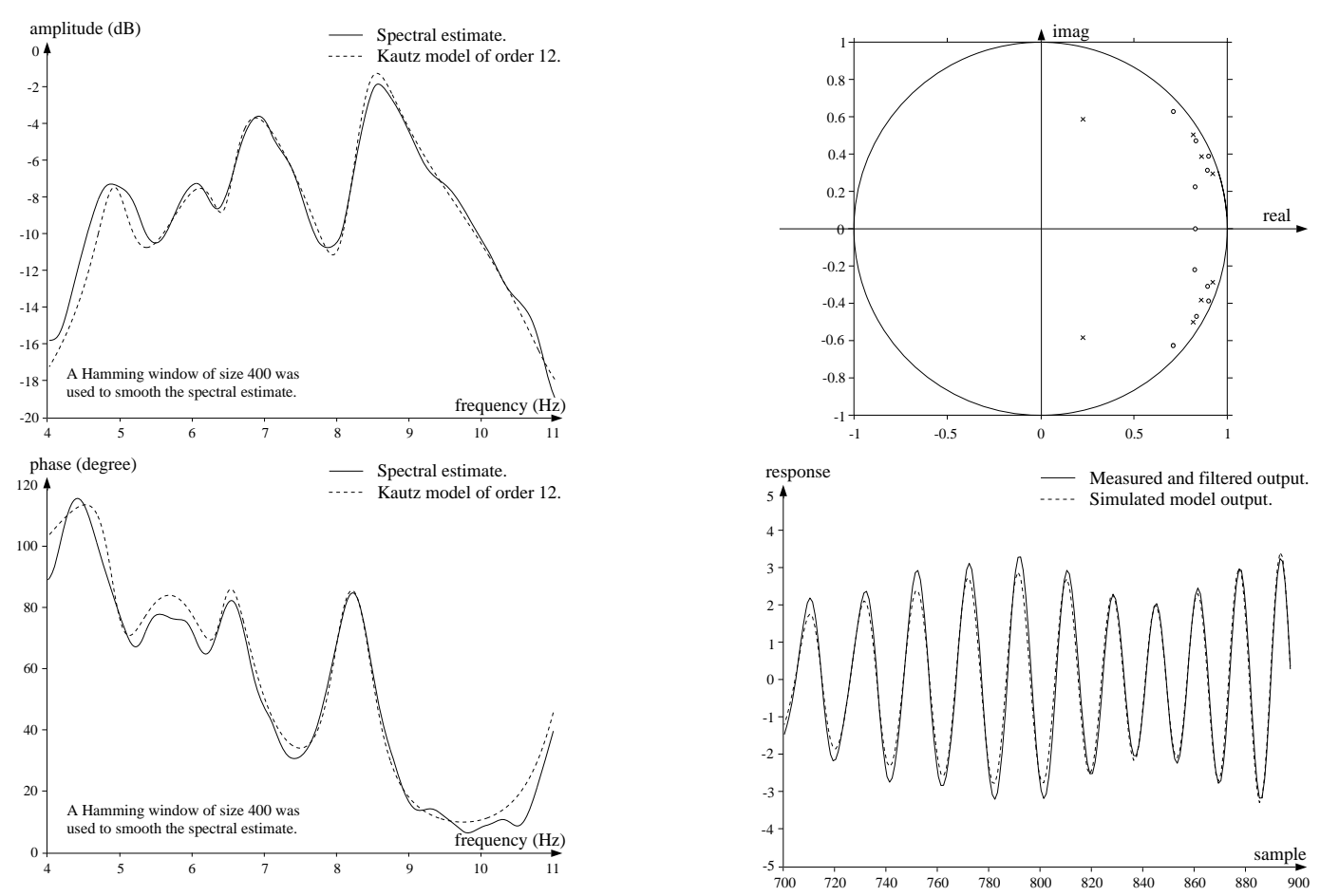

Fig. 3. Bode plot of the twelfth order Kautz model together with the spectral analysis estimate.

Finally, it should be remarked that the frequency response of the Kautz model of order 12 is very similar to the one obtained in Schoukens and Pintelon (1991) using the ELiS method. Yet the number of estimated parameters is larger (22) in the latter case.

\section{PROGRAM PACKAGE}

To extend and simplify the use of Laguerre and Kautz models in system identification a package of MATLAB m-files has been developed. The package is designed to utilize the features provided by the System Identification Toolbox (Ljung, 1991) and is freely available on request.

\section{CONCLUSION}

System identification using Kautz models has been applied to aircraft flight flutter data. Traditional models (ARX, OE and ARMAX) require high model orders to cope with the resonant behavior and low signal to noise ratio of the data. The relative short experimental time also limits the number of parameters that can be accurately estimated. The idea of Kautz models is to use a priori knowledge about the time constants and damping factors of the system to reduce the model complexity. It is shown that the flutter data can be accurately described by a low-order Kautz model,

whereas ARX, OE, and ARMAX models fail to describe the peaks in the data.

\section{ACKNOWLEDGMENT}

The authors gratefully thanks J. Schoukens and R. Pintelon for providing and describing the aircraft flight flutter data.

\section{REFERENCES}

Ljung, L. (1991). System Identification Toolbox User's Guide. The MathWorks, inc., Sherborn, MA.

Schoukens, J. and Pintelon, R. (1991). Identification of Linear Systems. A Practical Guideline to Accurate Modeling. Pergamon Press, U.K.

Wahlberg, B. (1991a). "Identification of resonant systems using Kautz filters". In Proc. of the 30th CDC, pages 2005-2010, Brighton, U.K.

Wahlberg, B. (1991b). "System identification using Laguerre models". IEEE Trans. on Automatic Control, AC-36(5):551-562. 